\title{
Off-Axis Parabolic Optical Relays: Almost Perfect Imaging
}

\author{
John L. Holdsworth*a ${ }^{\mathrm{a}}$, Galiya Sharafutdinova ${ }^{\mathrm{a}}$, Michael J. Sanderson $^{\mathrm{b}}$, Dirk F. van Helden ${ }^{\mathrm{a}}$, \\ a \\ University of Newcastle, University Drive, Callaghan, NSW 2308 Australia; \\ b \\ Department of Physiology, University of Massachusetts Medical School \\ 55 Lake Ave North Worcester, MA, 01655 USA
}

\begin{abstract}
Off-axis parabolic elements have found application in Tera-Hertz imaging and new application in two-photon microscopy scan engines. Both these applications demand spatial and temporal precision in photon location. This modelling work examines how close off-axis parabolic elements are to the perfect image relay by calculating the geometrical performance metrics of spot diagrams, point spread functions and wavefront distortion for different arrangements of off-axis parabolas and a parabolic torus when compared to a close-coupled two mirror scan engine. Results identify the off-axis parabolic elements as a superior scan engine particularly in wide-field scan instruments. When within the optimum beam size versus parabola focus length ratio, the imaging of these systems is superb.
\end{abstract}

Keywords: Off-axis parabolic reflectors, afocal relay, scanning.

\section{INTRODUCTION}

The discovery and naming of the parabola are attributed to Greek mathematicians Menaechmus (380BC-320BC) and Apollonius (262BC-190BC) with the definition of the properties by Pappus (290-350) while Newton examined the optical focussing properties of a parabolic surface. With the passage of centuries, these surfaces are still under study and finding new applications directing light. The ability of a parabolic reflecting surface to focus collimated light incident parallel to the optical axis to a focal point with minimal aberration has seen the surface be the choice for astronomical optics and, on a very much smaller scale, an off-axis segment being used to either focus light to an unobstructed focal point, or accept and collimate light from a focal point. The alternative approach of tilting spherical optics introduces spherical aberrations and astigmatism that will degrade the image properties and distort wavefronts.

The advent of the femtosecond pulsed laser has fostered two applications in particular that require superb wavefront preservation in both spatial and temporal dimensions. There is a body of work in Tera-Hertz imaging ${ }^{1-4}$ that has initiated and characterised the performance of a two mirror optical relay formed from dual off-axis parabolic (OAP) reflectors as shown in Figure 1.

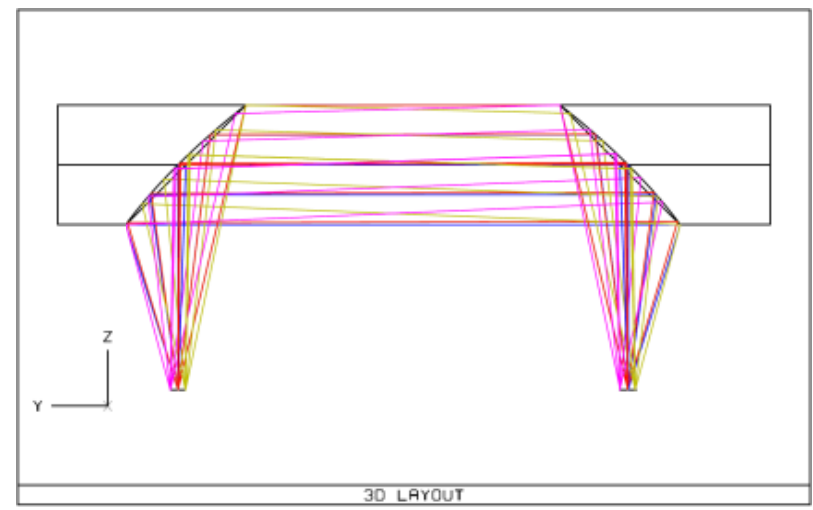

Figure 1. Dual off-axis parabolic elements arranged for THZ imaging. ${ }^{3}$.

22nd Congress of the International Commission for Optics: Light for the Development of the World, edited by Ramón Rodríguez-Vera, Rufino Díaz-Uribe, Proc. of SPIE Vol. 8011, 80112D

2011 SPIE · CCC code: $0277-786$ X/11/\$18 - doi: $10.1117 / 12.903430$

Proc. of SPIE Vol. 8011 80112D-1 
Ding and co-workers ${ }^{5}$ developed a reflective $\mathrm{THz}$ imaging system based on a combination of five OAP elements as shown in Figure 2.

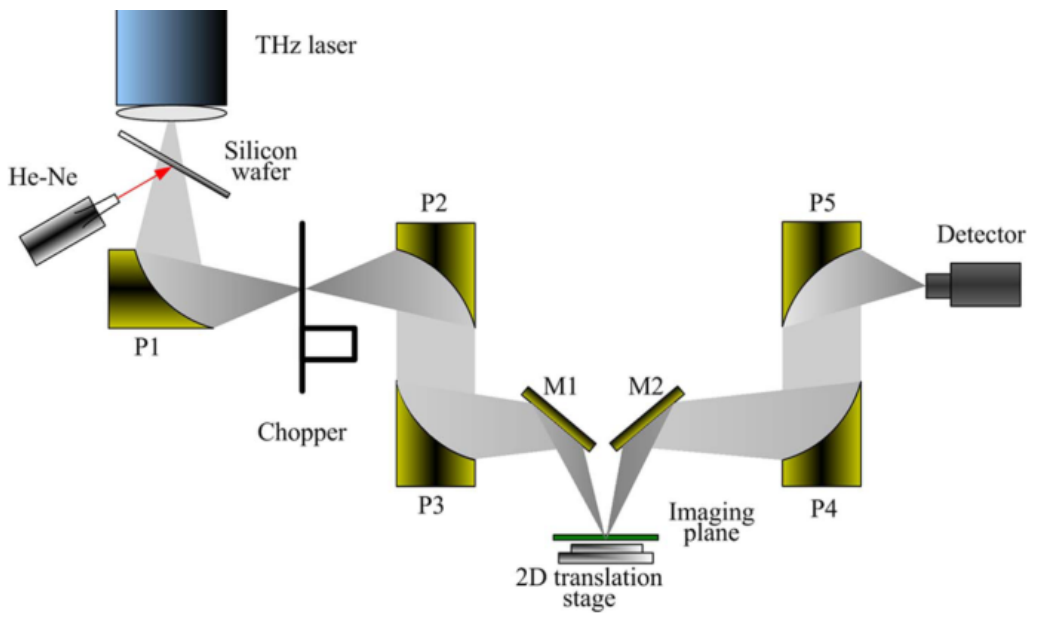

Figure 2. Imaging relay incorporating five off-axis parabolic elements arranged for THZ imaging ${ }^{5}$.

The optical performance of these systems is paramount as they relay femtosecond induced $\mathrm{THz}$ frequency radiation to and from samples. However the systems do have imperfections. The OAP surface P1 in Figure 2 above is focussing light from a point source to a point focus and that is not what Pappus originally defined as a property of a parabola. Two-photon laser scanning microscopy is the other application where the OAP is applied as an afocal relay. One layout is shown in Figure 3 below, the Parabolic Scan Engine ${ }^{6}$ (PSE) and a common Close Coupled Mirror Engine (CCE).

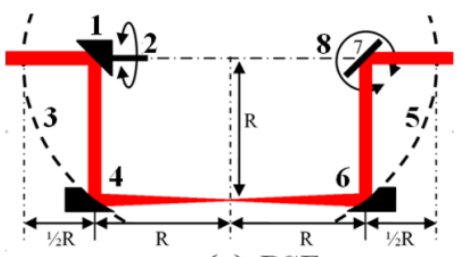

(a) PSE

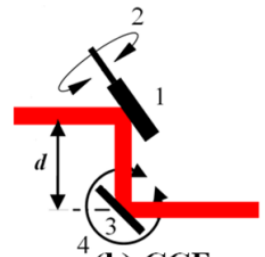

(b) $\mathrm{CCE}$

Figure 3. (a) Scanning afocal relay incorporating two off-axis parabolic elements and two scan mirrors and (b) comparative scan engine based on close coupled mirrors ${ }^{6}$.

A close look at this PSE design shows the scan mirrors positioned at the focal points of the first and second parabolic surfaces respectively and it is only the axial ray that passes through the focal point of both parabolic surfaces, again a departure from the elegant purity of Greek geometry. When considering real beams, that is non-zero beam widths and non-zero divergence, impinging upon three dimensional OAP reflectors, the inherent imperfections of the optical relays are uncovered.

The off-axis part of the rotationally symmetrical paraboloid is an asymmetric surface and the rays of a 2D incident beam are reflected from two perpendicular cross-sections; one of which is the 2D parabolic cross-section and the other is a circular surface of continuously variable decreasing radii towards the paraboloid vertex. This complexity is inherent in any particular OAP segment and is the reason for geometrical aberrations, astigmatism and coma in the resulting image. As in any common spherical profile optical system, a diffraction-limited image can be achieved only for paraxial rays parallel to the optical axis. The OAP surface is defined by the displacement relative to the focal point of the parent parabola and apparent off-axis focal length. Different segments have found different applications and high quality optics are available from suppliers e,g, Space Optics Research Labs MA USA. The 90 degree off-axis element is arguably the most useful in imaging and has the focal length equal to the radius of curvature of the parabolic surface. Replicated OAP optics are available from a number of suppliers e.g. Newport Corp. CA USA and Edmund Optics Inc. Singapore. The optical relay including two 90 degree off-axis elements has been found to preserve beam quality ${ }^{3}$ and can be used in scan systems ${ }^{6,7}$.

There are other known scanner designs with concave surfaces, both OAP surfaces and spherical reflectors, as well as only flat mirror scanners. In any discussion of properties, it is beneficial to compare results across designs. Aberrations introduced by the relay can destroy the propagating beam properties and it is especially important for two- and multi- 
photon microscopy that the ultra-short pulsed infra-red laser beam used as an excitation source have the temporal and spatial parameters preserved.

This research investigates the performance via modelling using OSLO optical software, Lambda Research Corporation, of 90 degree off-axis segments of various parabolic reflectors for the 2D scan engine application. Models are evaluated both as focused and afocal relays in terms of spot diagrams, the point spread functions (PSF), wavefront distortions and aberrations.

\section{METHOD}

Three designs, as shown in Figure 4, for afocal 2D scan relays formed from OAP elements are compared. Schematics (a) and (b) feature regular 3D parabolic surfaces, while schematic (c) is a parabolic torus, rotationally symmetric about the dotted axis and therefore circular in any cross section perpendicular to the page as patented by Seel ${ }^{8}$.

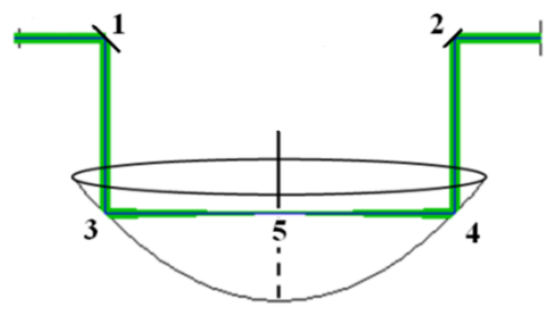

(a)

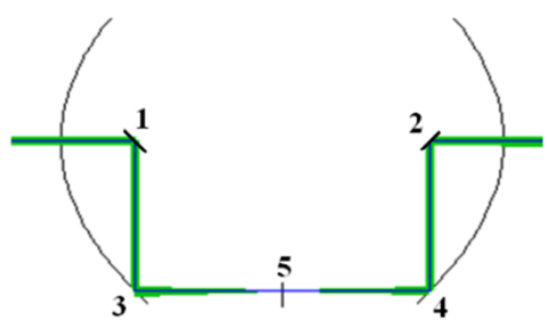

(b)

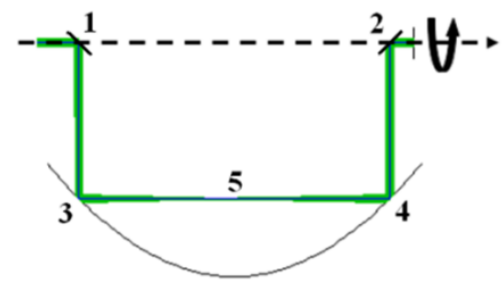

(c)

Figure 4. Three afocal relays with off-axis parabolic elements for $2 D$ scanning. (a) and (b) employ two $90^{\circ}$ off-axis segments of regular 3D parabolic surfaces with different orientation. (c) is a parabolic torus. Mirror 1 rotates about the input optical axis and mirror 2 rotates orthogonally perpendicular to the page. Reflections at 3 and 4 are off the segments of OAP or parabolic torus surfaces and 5 marks the focal point of light reflected from 3.

The performance of OAP surface 3 at the focal point 5 is compared for all three designs. The parabola radius initially chosen was $100 \mathrm{~mm}$. Recent modelling has varied the ratio of the parabola focal length dimension to beam size as developing a miniaturised device is attractive. The $90^{\circ} \mathrm{OAP}$ elements have effective focal length equal to the radius of curvature, so this value divided by the fixed entrance beam diameter provides the f-number. For this investigation the illuminating source was simulated as a circular $790 \mathrm{~nm}$ wavelength laser beam with Gaussian intensity profile and an $1 / e^{2}$ waist radius of $0.4 \mathrm{~mm}$, a half-angle divergence of $0.629 \mathrm{mRad}$ and an M-squared factor of 1 . The source is positioned $2 \mathrm{~m}$ away from the first scanning mirror. The aperture stop is on the first scanning mirror surface. The image aperture radius is $10 \mathrm{~mm}$ at a distance of $15 \mathrm{~mm}$ away from the last scanning mirror. The aperture radius and distance were chosen to mimic the entrance aperture of a scan lens which usually follows a scan engine. The engines are analysed as afocal relay systems and with a scan lens. In all engine designs, the scanning mirror surfaces move $\pm 10^{\circ}$ in each single axis, producing a $\pm 20^{\circ}$ line scan about the original zero position. With the four mirror systems, the first mirror scans in the horizontal direction and the second mirror scans the vertical axis.

Twenty five scan field points including zero, $5^{\circ}$, and maximum deflection $10^{\circ}$ in each direction and both axes were defined and these have been used as the basis for calculations representing the complete frame in a scan pattern.

\section{RESULTS AND DISCUSSIONS}

\subsection{The focal point performance}

Investigating the focusing performance of a single $90^{\circ}$ off-axis parabolic reflector, for light incident parallel to the optical axis, shows the presence of aberrations at the focal point. Four spot diagrams for varying incident beam sizes and radii of curvature are shown in Figure 5 showing coma and astigmatism at the focal point. The results demonstrate that for particular incident beam size and radius of curvature the aberrations can destroy the diffraction-limited performance at the focal point. The ratio between the incident beam diameter and the focal length of the OAP element, which is it's radius of curvature, is equivalent to the spherical optic $\mathrm{f} / \#$ and to achieve the best possible diffraction-limited performance at the focal point the radius of curvature should be more than 15 times beam diameter. 


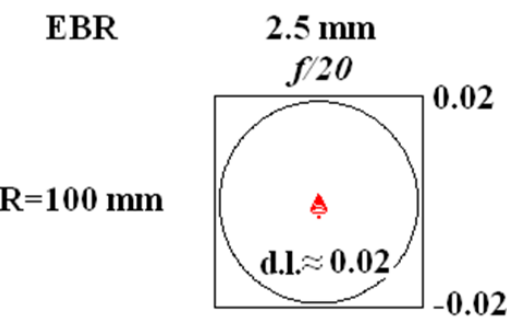

(a)
$5 \mathrm{~mm}$

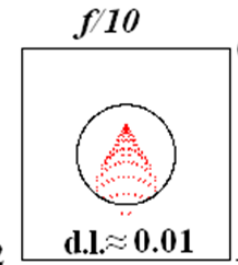

(b)

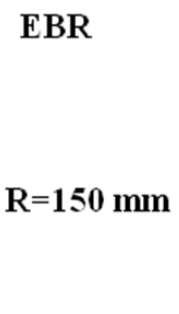

0.02

\section{2}

0.02

(c)

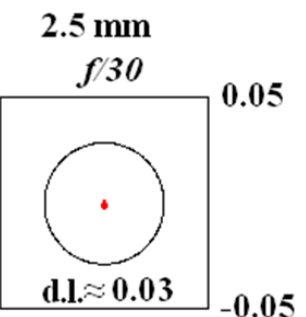

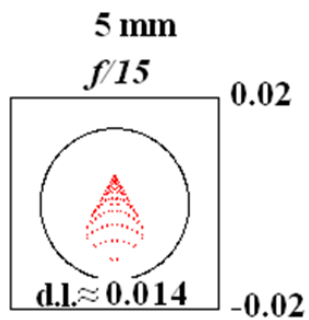

(d)

Figure 5. Focal point spot diagrams for a regular OAP for collimated light incident parallel to the optical axis of parental parabola. The black circle inside each square represents an Airy disk illustrating diffraction-limited performance and with the radius shown. The OAP radius of curvature is $100 \mathrm{~mm}$ for panels (a) and (b) and $150 \mathrm{~mm}$ for panels (c) and (d). The entrance beam radius is $2.5 \mathrm{~mm}$ for (a) and (c), and $5 \mathrm{~mm}$ for (b) and (d). The f-number is calculated from the ratio of focal length to the beam diameter.

The spot diagrams and aberration at the focal point of a reversed $90^{\circ}$ OAP reflector i.e. as in Figure 4 (b) when light impinges from the focal point, are shown in Figure 6. The results shows that introduced aberrations are similar to the aberration at the focal point of "normal" off-axis parabola for respective parameters of beam radius and the radius of curvature. From the spot diagrams it is clear coma dominates over all other aberrations. For diffraction-limited performance at the focal point the radius of curvature should be more than 25 times beam diameter.

For the $90^{\circ}$ off-axis segment of the parabolic torus, i.e. as in Figure 4 (c), the spot diagrams at the focal point are presented in Figure 7. There is a different contribution of five aberrations to the focal image. There is evidence of greater spherical aberration in reflection by the parabolic torus and due to this there is a better centre of mass of the rays than in the rotationally symmetrical parabolic reflector result of Figure 5. For diffraction-limited performance at the focal point the radius of curvature should be more than 25 times beam diameter.

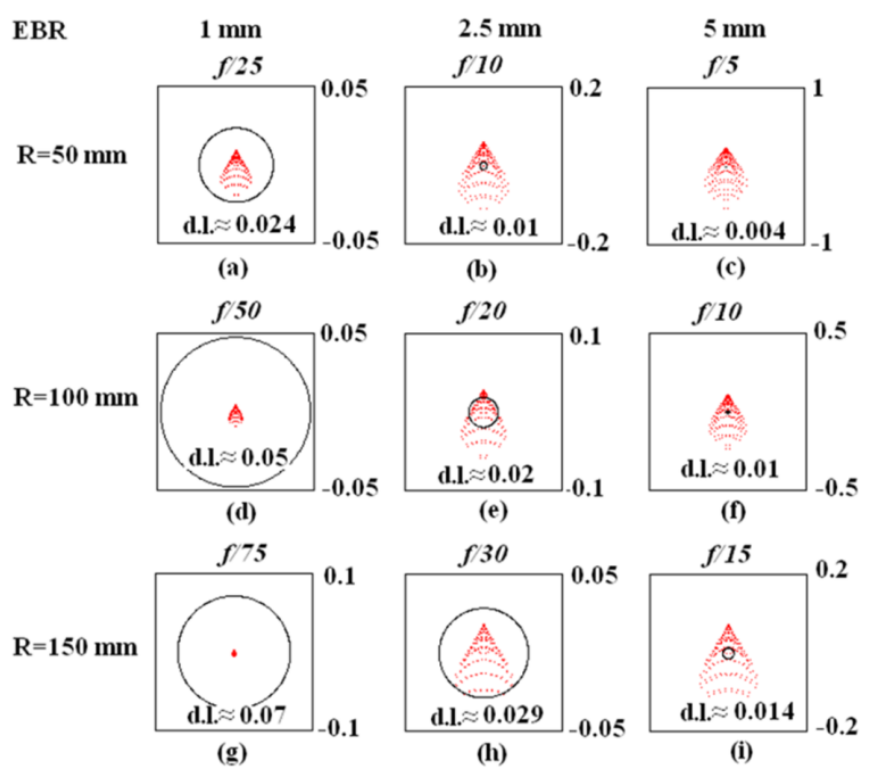

Figure 6. Spot diagrams at the focal point of $90^{\circ}$ off-axis segment of symmetrical parabola for light incident from the focal point of parental parabola. The black circle inside each square represents an Airy disk illustrating diffraction-limited performance and with the radius shown. OAP radius of curvature: $50 \mathrm{~mm}$ for (a)-(c), $100 \mathrm{~mm}$ for (d)-(f); $150 \mathrm{~mm}$ for (g)-(i). The entrance beam radius is: $1 \mathrm{~mm}$ for (a), (d) and (g); $2.5 \mathrm{~mm}$ for (b), (e) and (h); and $5 \mathrm{~mm}$ for (c), (f) and (i). The f-number is calculated from the ratio of focal length to the beam diameter. 

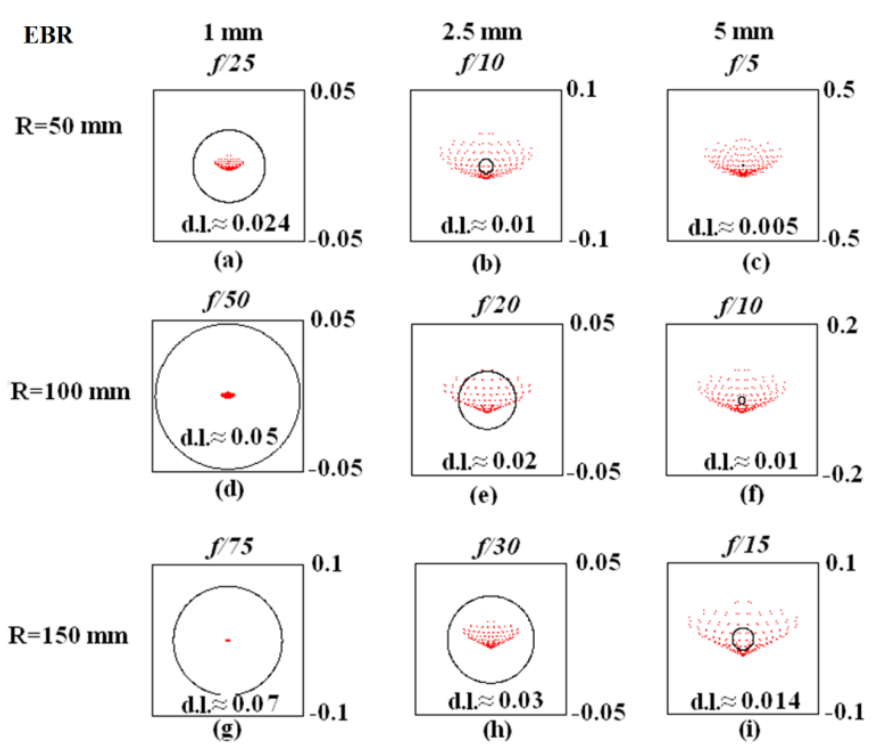

Figure 7. Spot diagrams at the focal point of $90^{\circ}$ off-axis segment of parabolic torus for the light incident parallel to the optical axis of parental parabola. Black circle inside each square presents an Airy disk and is illustrated to visualise the diffractionlimited performance. The radius of Airy disk is shown inside each square as d.l. (diffraction-limited size). At (a)-(c) radius of curvature is $50 \mathrm{~mm}$; at (d)-(f) radius of curvature is $100 \mathrm{~mm}$, and at (g)-(i) radius of curvature is $150 \mathrm{~mm}$. The entrance beam radius is $1 \mathrm{~mm}$ at (a), (d) and (g); $2.5 \mathrm{~mm}$ at (b), (e) and (h); and $5 \mathrm{~mm}$ at (c), (f) and (i). F-number is calculated as the focal distance relative to the beam diameter.

\subsection{Investigation of afocal parabolic reflector relays}

To build a 2D scanner it is necessary to produce a system deflecting from one-pivot point and of the designs in Figure 4, only two, the PSE and Seel designs, achieve this requirement. The mirror arrangement and the deflected rays are shown in Figure 8 detailing the single deflection point achieved. In comparison, the "normal" parabolic surface with deflecting scan mirrors in Figure 9 does not meet the requirement for the rays to impinge at one point on the second scan mirror.

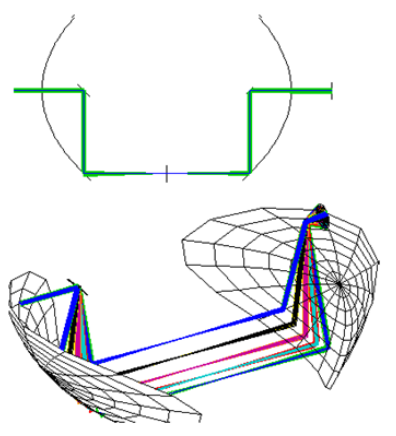

(a)

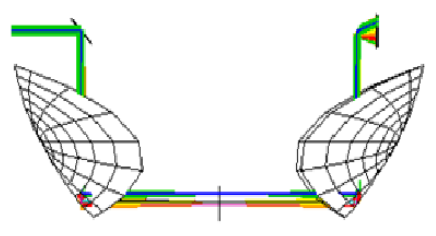

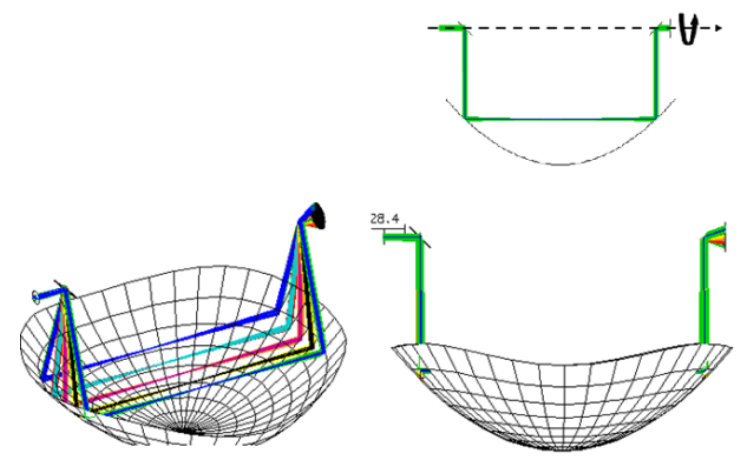

(b)

Figure 8. Afocal scan relays (a) with off-axis parabolic elements (PSE) and (b) a parabolic torus (Seel). Scan designs and lens drawing for 25 scanning positions. 


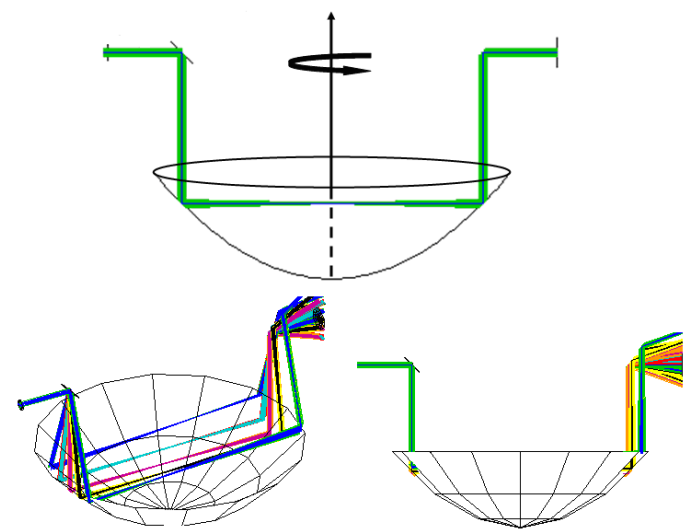

Figure 9. Afocal scan relay with off-axis parabolic elements using rotationally symmetrical parabolic surfaces. Scan engine design and lens drawing for 25 scanning positions showing the lack of effective single effective deflection point.

The foot print, spot diagrams and point spread function (PSF) in Figure show the correction that results from the second reflector where these data are at an f/\# of 20. Row (a) in Figure shows the footprint pattern across the scan field, row (b) shows the spot diagrams with slight differences in the shape and centre of mass between two relays and row (c) shows the PSF images of the on-axis rays. The nearly uniform intensity distribution across the spots is an indication of correction and the elongation at the periphery of the scan can be computed in terms of an effective temporal distortion induced by the scan engine.

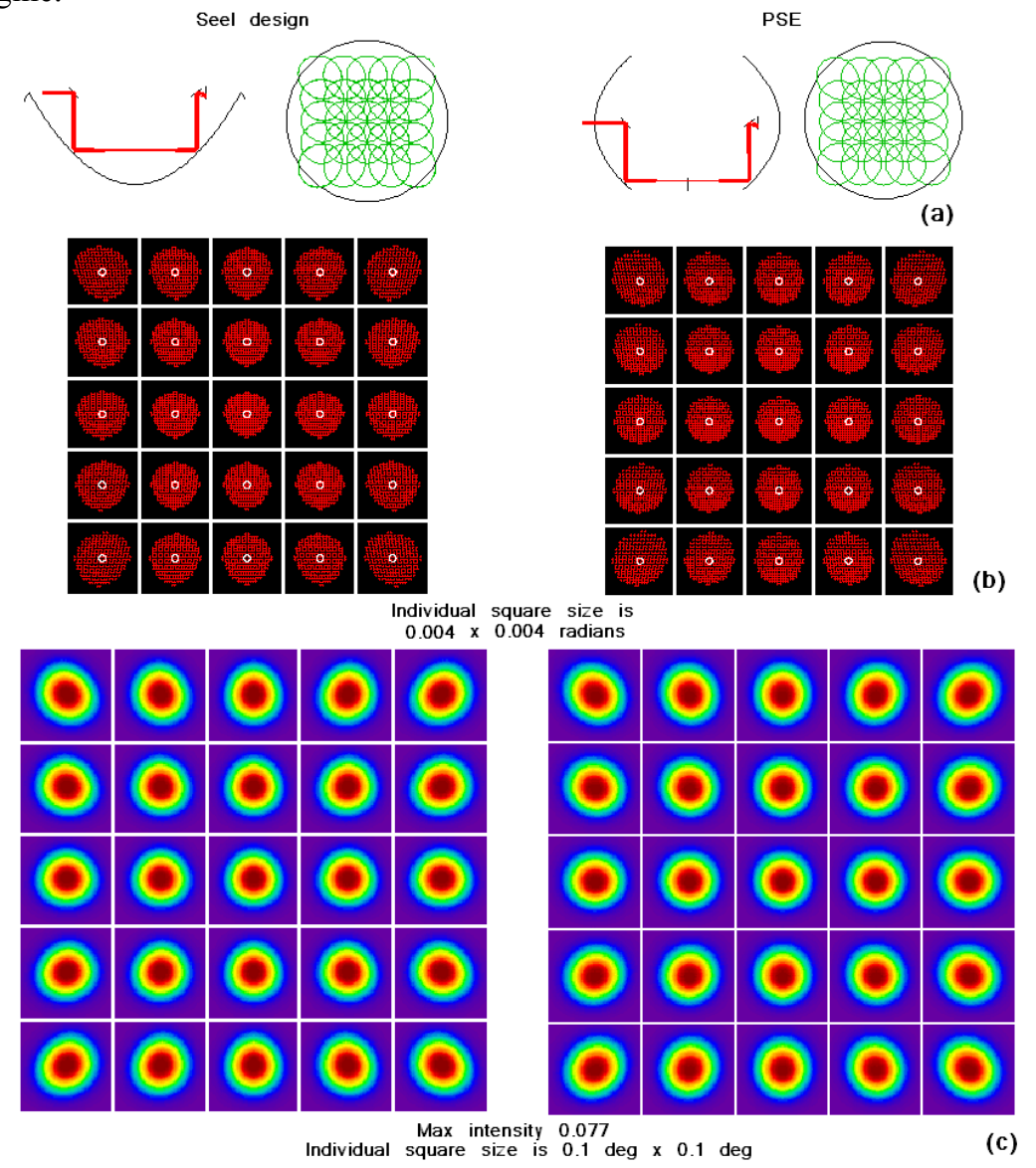

Figure 10. Comparative performance at 25 points across the two dimensional $\pm 10^{\circ}$ scan field for the Seel design and the PSE. Row (a) illustrates the simulated lens drawing and the image of 25 spots at the image surface. Row (b) shows the spot diagrams and row (c) shows PSFs for the 25 scan points. 
A scan engine interfacing to a microscope does so through a scan lens. In order to compare the relative merit of three engines, the PSE, the Seel and the close coupled mirror (CCE) designs, the operation of the three engines over a central \pm $5^{\circ}$ scan field was studied after focussing to an intermediate plane through an optimised scan lens. The scan lens does introduce mild aberrations however this is the only path to a true comparison of performance. The results displayed in Figure 11 are of great interest when one brings in the temporal requirements of two-photon scanning. The greater the uniformity and flatness of the optical path, the better the generated signal within the excited sample volume. These results are greatly encouraging for the PSE design and work has commenced on the experimental verification of the scan engine. Further, the uniformity over a wide field is unparalleled when compared the CCE design used in current commercial systems.

PSE
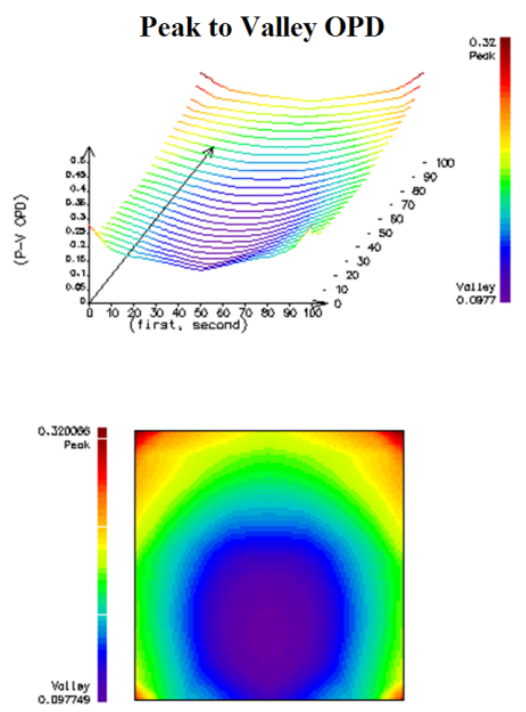

(a)
Seel
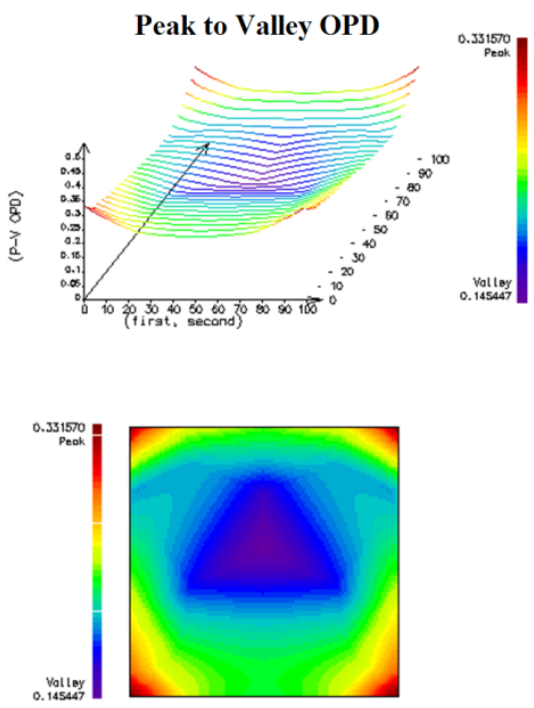

(b)
CCE
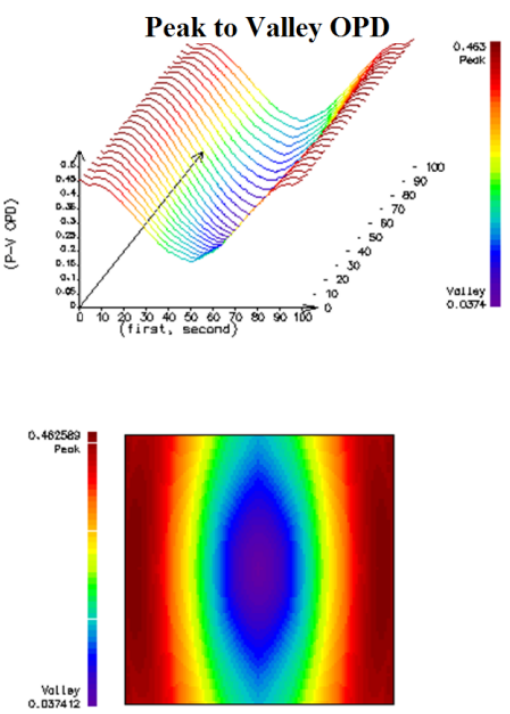

(c)

Figure 11. Comparative optical path difference performance across a two dimensional $\pm 5^{\circ}$ scan field for (a) the PSE, (b) the Seel design and (c) the CCE. Output scales are in units of wavelength.

\subsection{Experimental realisation}

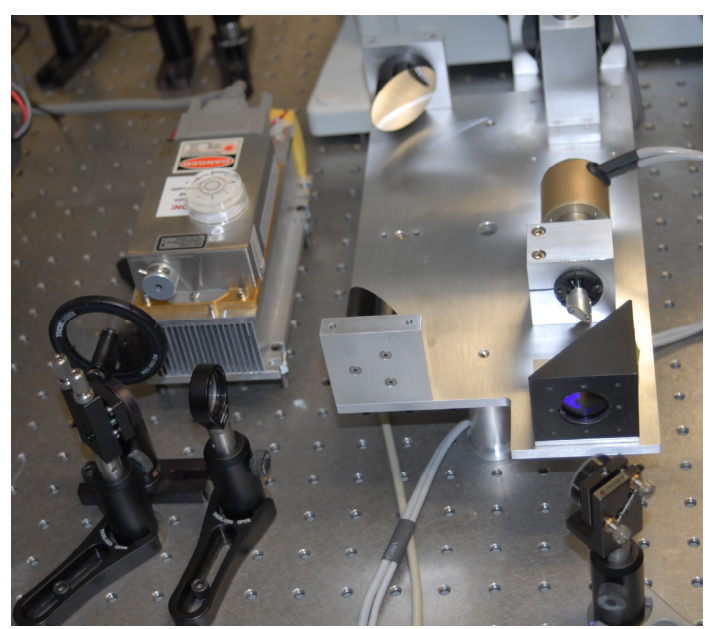

(a)

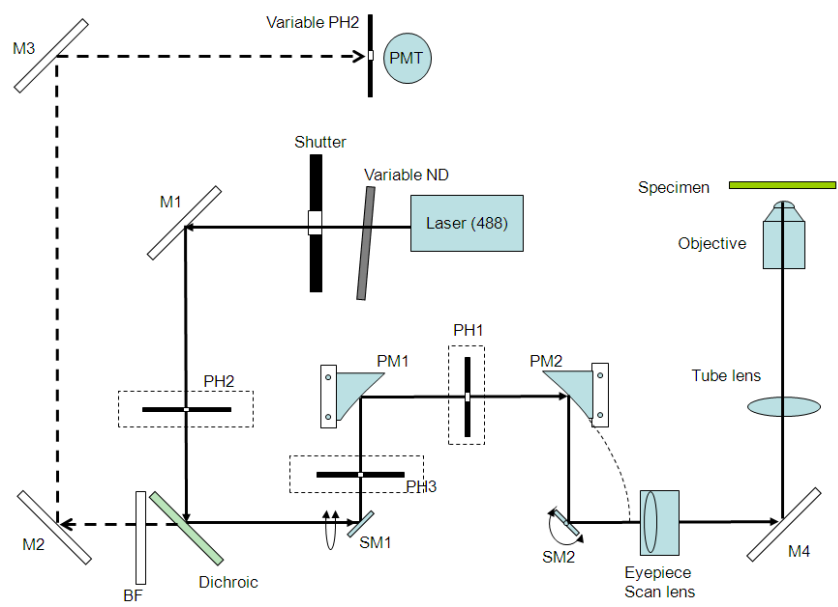

(b)

Figure 12. (a) Plate of prototype and (b) optical schematic configured for single photon microscopy. 
A prototype PSE scanner has been constructed (Figure 12) in the Sanderson laboratory and has been used to generate images both as a confocal single photon scanning microscope and as a two photon microscope. The images produced in both single and dual photon modes are qualitatively good when compared to images obtained in commercial systems and work is continuing to improve the optical quality and to establish a basis of comparison between like images obtained in different systems.

\section{SUMMARY}

The focusing performance of off-axis sections for three different parabolic reflecting surfaces has been evaluated and shows the presence of aberrations emerging from the OAP surfaces. There is a loss of performance when critical $\mathrm{f} / \#$ values are exceeded for each OAP configuration studied. The use as an optical relay with scanning mirrors restricts the choice of OAP relays to two and the Seel relay is an expensive element to fabricate. The wavefront and temporal behaviour of the PSE system is exemplary and the development and testing of a prototype PSE scanner gives hope that the almost perfect imaging of the OAP will translate to spectacular microscopy.

\section{ACKNOWLEDGEMENT}

Galiya Sharafutdinova acknowledges the University of Newcastle, Australia, for stipend support.

\section{REFERENCES}

[1]. Arguijo, P., and Scholl, M. S., "Exact ray-trace beam for an off-axis paraboloid surface". Applied Optics, 42(16), 3284-3289, (2003).

[2]. Arguijo, P., Scholl, M. S., and Paez, G., "Diffraction patterns formed by an off-axis paraboloid surface." Applied Optics, 40(17), 2909-2916, (2001)

[3]. Brückner, C., Notni, G., and Tünnermann, A. "Optimal arrangement of $90^{\circ}$ off-axis parabolic mirrors in $\mathrm{THz}$ setups." Optik, 121, 113-119, (2010).

[4]. Hu, B., and Nuss, M. "Imaging with THz waves." Optics Letters, 20(16), 1716-1720, (1995).

[5]. Ding, S.-H., Li, Q., Yau, R., and Wang, Q. "High-resolution terahertz reflective imaging and image resoration." Applied Optics, 49(36), 6834-6839, (2010).

[6]. Sharafutdinova, G., Holdsworth, J., and van Helden, D. "Improved field scanner incorporating parabolic optics. Part 1: Simulation." Applied Optics, 48(22), 4389-4396, (2009)

[7]. Sharafutdinova, G., Holdsworth, J., and van Helden, D., "Improved field scanner incorporating parabolic optics. Part 2: experimental verification and potential for volume scanning." Applied Optics, 49(29), 5517-5527 (2010).

[8]. Seel, M. Patent No. US6,433,908. USA. (2002). 